\title{
EDITORIAL
}

\section{Does "A" stand for alveolization?}

\author{
J.S. Torday, V.K. Rehan
}

The current issue of the European Respiratory Journal (ERJ) publishes a report by HIND and MADEN [1] showing vitamin A induction of alveolization which has been read with great interest. Vitamin $\mathrm{A}$ is a nutrient that is important for lung organogenesis, homeostasis and repair. It was more than 60 years ago that WOLBACH and HowE [2] first demonstrated that vitamin A deprivation caused squamous metaplasia of conducting airway epithelium, that could be reversed by vitamin A repletion. Since this report many clinical studies and animal models, have supported the importance of dietary retinoids in lung morphogenesis and repair. These studies have generated optimism that retinoic acid might be useful for promoting alveolization in both the premature neonatal and emphysematous adult lung. Premature infants who are at risk of developing bronchopulmonary dysplasia (BPD) have low retinoid liver stores and blood levels at birth [3, 4]. In fact, infants who develop BPD show squamous metaplasia of the conducting airways and show alveolar arrest, findings similar to those occurring in vitamin A-depleted animals. Therefore, it is not surprising that several investigators suggest that the course of BPD in very low birth-weight infants can be ameliorated by supplemental vitamin A $[5,6]$. In a randomised, controlled multicentre clinical trial, vitamin A supplementation caused a small, but significant reduction in BPD [7].

The involvement of vitamin A, not only as a preventive but also as a therapeutic role in lung injury, has previously been suggested by many investigators. For example, MASSARO and MASSARO [8] showed that in rat pups whose alveolization process had been arrested by exogenous corticosteroids, retinoic acid administration caused a dramatic increase in the number of alveoli, through the proliferation of secondary septa. The studies by HIND and MADEN [1], reported in this issue extend the work by MASSARo and MASSARo [8] and their own previous work, by showing that in a mouse model firstly that disulphiram, a retinoic acid (RA) synthesis inhibitor, disrupts alveolar development, and secondly that RA, when administered to adult mice, restores the lung cytoarchitecture that had previously been disrupted by the postnatal administration of dexamethasone.

Although it is becoming clear that retinoic acid, a biologically active derivative of vitamin A, plays an important role in both the biochemical (surfactant) and structural (alveolization) maturation of the developing lung, the molecular mechanisms involved remain poorly understood. In fact, the molecular signals responsible for the normal initiation of alveolization and its developmental regulation are largely unknown. However, the following lines of observation suggest that retinoids play a key regulatory role in this process. These

Harbor-UCLA Medical Center, Research and Education Institute, Torrance, California, USA.

Correspondence: J.S. Torday, Harbor-UCLA Medical Center, Research and Education Institute, Torrance, California, USA. Fax: 1310222 3887. E-mail: jtorday@gcrc.rei.edu include: 1) the abundant presence of retinol-rich lipofibroblasts in the alveolar wall during the period of normal septation, and their significant diminution after septation has been completed [9]; 2) high concentrations in the lung of RA synthesising enzymes, receptors, and cytoplasmic proteins during the period of septation [8];3) dexamethasone-induced inhibition of septation is associated with a diminution in the concentration of cellular retinol binding protein (CRBP)-I messenger ribonucleic acid (mRNA) and up-regulation of CRBP-I mRNA on treatment with RA [10]. More direct evidence that RA regulates septation is shown by the following: 1) mice made mutant for RA receptor-gamma, fail to form alveoli normally [11]; 2) RA treatment of neonatal rats during the period of spontaneous septation causes a $50 \%$ increase in alveolar number without an increase in alveolar volume [7]; 3) dexamethasone treatment during the period of spontaneous alveolization arrests this process and RA treatment during this period prevents the dexamethasone-induced inhibition of alveolization [7]; 4) RA treatment of adult rats, previously made emphysematous by the intratracheal instillation of elastase, causes the induction of septation [12]; 5) RA induces septation in foetal mouse lung explants; 6) treatment of prematurely delivered sheep with retinol increases alveolar number; and 7) the current work of HIND and MADEN [1] can be added to this growing list for the evidence of RA involvement in alveolization. However, more work has to be done and many questions need to be answered before these findings can be extrapolated to retinoic acid for human studies. As acknowledged by HIND and MADEN [1] not all studies have shown that retinoic acid can rescue alveolar regeneration in all lung injury models. Some have shown only a partial rescue of alveolar surface area in elastase-treated rats $[13,14]$ and some have, in fact, reported no effect at all [15, 16]. More importantly, almost no data are available to assess the functional capacity of the rescued lung. It is crucial that these measurements are made both at the cellular (e.g. surfactant synthesis by type II cells and lipid uptake by the lipofibroblasts) and at the whole animal level (ventilation/ perfusion matching) before these studies can be extended to humans. In fact, rather disappointingly, when lung function was assessed in a rat model, no effect could be demonstrated with RA treatment [15].

The exact role of RA in alveologenesis and the underlying molecular mechanisms involved, need to be clearly elucidated. It is important to point out that retinoids may regulate only the eruption of septa and their spacing (the distance between the septa), and not the septal length, and therefore not the ultimate alveolar size, which are probably determined by other regulators that provide feedback inhibition of septal elongation when optimal alveolar size has been attained. This is supported by previous findings [8] in a dexamethasoneinduced lung injury rat model that RA only increased alveolar number without increasing alveolar size/volume (gas-exchanging area). Understanding how retinoids influence the sites of secondary septal sprouting is fundamental to the process of understanding alveolization [17]. Whether retinoids modulate 
pre-programmed sites for secondary septum formation or provide a morphogenetic gradient for chosen sites for septal formation needs to be explored. Or retinoids might work by recruiting either dormant or circulating stem cells to sites of septal sprouting during the process of alveolization. The whole array of retinoid-responsive genes, their temporal and spatial expression, their regulators (including coactivators and corepressors), and species-specific information is now needed to take the retinoid story to the next level.

Recent studies of the role of interstitial fibroblasts in lung morphogenesis, homeostasis and pathogenesis [18] may provide clues to how retinoic acid determines septation of the lung alveolus-lipofibroblasts, which "traffick" the lipid inclusions that retinoids are stored in [19], are strategically positioned beneath alveolar type II cells [20], and can transdifferentiate to myofibroblasts, the cellular core of the alveolar septum [21]. Further understanding of the spatial and temporal determinants of the lipo- and myofibroblast will reveal the mechanisms of alveolar wall septation, remodelling and regeneration. Using focused technologies such as in situ hydridisation, laser capture microdissection coupled with micro-arrays on these samples, and confocal microscopy should be used to sort out the key players from a large number of retinoid-responsive genes before a great deal of progress can be made for retinoids to serve as preventive and/ or therapeutic modalities for human lung disease. Until these questions are answered retinoic acid intervention is likely to be limited only to specific experimental models of lung injury that specifically involve disruption of retinoic acid-mediated pathways, e.g., disulphiram- or dexamethasone-induced lung damage rather than a "magic bullet" for many other forms of lung injury. Even then, a large amount of work needs to be performed to explore the effects of therapeutic doses of retinoid administration on other organ systems, including the developing heart and brain, before we can "take the 'A' train" safely.

\section{References}

1. Hind M, Maden M. Retinoic acid induces alveolar regeneration in the adult mouse lung. Eur Respir J 2004; 23: 20-27.

2. Wolbach SB, Howe PR. Epithelial repair in recovery from vitamin A deficiency: an experimental study. $J$ Exp Med 1933; 57: 511-526.

3. Shenai JP, Chytil F, Jhaveri A, Stahlman MT. Plasma vitamin $\mathrm{A}$ and retinol-binding protein in premature and term neonates. J Pediat 1981; 99: 302-305.

4. Shenai JP, Chytil F, Stahlman MT. Liver vitamin A reserves of very low birth weight neonates. Pediatr Res 1985; 19: 892893.

5. Shenai JP, Kennedy KA, Chytil F, Stahlman MT. Clinical trial of vitamin A supplementation in infants susceptible to bronchopulmonary dysplasia. J Pediatr 1987; 111: 269-277.

6. Robbins ST, Fletcher AB. Early vs delayed vitamin A supplementation in very-low-birth-weight infants. JPEN 1993; 17: 220-225.

7. Pearson E, Bose C, Snidow T, et al. Trial of vitamin A supplementation in very low birth weight infants at risk for bronchopulmonary dysplasia. J Pediatr 1992; 121: 420-427.

8. Massaro GD, Massaro D. Postnatal treatment with retinoic acid increases the number of pulmonary alveoli in rats. $\mathrm{Am}$ J Physiol (Lung Cell Mol Physiol) 1996; 270: L305-L310.

9. Vaccaro C, Brody JS. Ultrastructure of developing alveoli. I. The role of the interstitial fibroblast. Anat Rec 1978; 192: 467-479.

10. Rush MG, Ul-Haq R, Chytil F. Opposing effects of retinoic acid and dexamethasone on cellular retinol-binding protein ribonucleic acid levels in the rat. Endocrinology 1991; 129: 705-709.

11. McGowan SE, Jackson SK, Jenkins-Moore M, Dai HH, Chambon P, Snyder JM. Mice bearing deletions of retinoic acid receptors demonstrate reduced lung elastin and alveolar numbers. Am J Respi Cell Mol Biol 2000; 23: 162-167.

12. Massaro GD, Massaro D. Retinoic acid treatment abrogates elastase-induced pulmonary emphysema in rats. Nat Med 1997; 3: 675-677.

13. Belloni PN, Garvin L, Mao CP, Bailey-Healy I, Leaffer D. Effects of all-trans-retinoic acid in promoting alveolar repair. Chest 2000; 117: 235S-241S.

14. Tepper J, Pfeiffer J, Aldrich $\mathrm{M}$, et al. Can retinoic acid ameliorate the physiologic and morphologic effects of elastase instillation in the rat? Chest 2000; 117: Suppl. 1, 242S-244S.

15. Srinivasan G, Bruce EN, Houtz PK, Bruce MC. Dexamethasone-induced changes in lung function are not prevented by concomitant treatment with retinoic acid. $\mathrm{Am}$ J Physiol Lung Cell Mol Physiol 2002; 283: L275-L287.

16. Lucey EC, Goldstein RH, Breuer R, Rexer BN, Ong DE, Snider GL. Retinoic acid does not affect alveolar septation in adult FVB mice with elastase-induced emphysema. Respiration 2003; 70: 200-205.

17. Pierce RA, Michael Shipley J. Retinoid-enhanced alveolization: identifying relevant downstream targets. Am J Respir Cell Mol Biol 2000; 23: 137-141.

18. Torday JS, Rehan VK. Mechanotransduction determines the structure and function of lung and bone: A theoretical model for the pathophysiology of chronic disease. Cell Biochem Biophys 2003; 37: 235-246.

19. Schultz CJ, Londos C, Sun H, Torres E, Torday JS. Role of adipocyte differentiation related protein in lung surfactant phospholipid synthesis. Am J Physiol Lung Cell Mol Physiol 2002; 283: L288-L296.

20. Gewolb IH, Torday JS. High glucose inhibits morphologic maturation of the foetal lung in vitro. Morphometric analysis of lamellar bodies and fibroblast lipid inclusions. Lab Invest 1995; 73: 59-63.

21. Torday JS, Torres E, Rehan VK. The role of fibroblast transdifferentiation in lung epithelial cell proliferation, differentiation, and repair in vitro. Pediatr Pathol Mol Med 2003; 22: 189-207. 